\title{
Comportamiento higrotérmico y energético en período estival de un edificio escolar típico en la ciudad de San Juan, Argentina
}

\section{Hygrothermal and energy performance in summer period of a typical school building in the city of San Juan, Argentina}

María Guillermina Ré * Instituto Regional de Planeamiento y Hábitat. Facultad de Arquitectura Urbanismo y Diseño. Universidad

Nacional de San Juan guillerminare@gmail.com

Irene Blasco Lucas ** Instituto Regional de Planeamiento y Hábitat. Facultad de Arquitectura Urbanismo y Diseño. Universidad Nacional de San Juan iblasco@faud.unsj.edu.ar

Celina Filippín *** Consejo Nacional de Investigaciones Cientificas y Técnicas cfilippin@cpenet.com.ar 


\section{Resumen}

El objetivo del trabajo es evaluar el comportamiento higrotérmico y energético en época estival de un edificio escolar representativo en la ciudad de San Juan, Argentina. Para ello, se procesan con PROMEDI-HTL las mediciones de irradiancia solar, temperatura y humedad, realizadas cada 15 minutos y en períodos de 15 a 20 días continuos en tres momentos claves de los años 2013-2014, bajo diferentes situaciones de uso. Dichos registros se relacionan con lecturas diarias de consumo eléctrico. Adicionalmente se estudian los niveles de confort en tres aulas mediante el método Fanger, determinando con un software específico los índices PMV (Valor Medio Predicho) y PPD (Porcentaje de Personas en Disconfort). Se analizan estadísticamente series mensuales correspondientes a nueve años de consumo eléctrico y se efectúa el cálculo térmico-energético estacionario aplicando las Normas IRAM mediante el modelo KG-MOD. Los valores empíricos se comparan con los teóricos obtenidos.

Palabras clave: Edificio escolar; Evaluación energética; Confort higrotérmico

\section{Abstract}

The objective of this work is to evaluate summer hygrothermal and energy performance of a representative school building in the city of San Juan, Argentina. To achieve it, solar irradiance, temperature and humidity measurements, made every 15 minutes during periods of 15 to 20 consecutive days in three key moments in the years 2013-2014 under different conditions of use, are processed with PROMEDI-HTL. These records are related to daily readings of electricity consumption. Additionally, comfort levels are studied in three classrooms using Fanger method to determine the PMV index (Predicted value Middle) and PPD (Percentage of Persons in Discomfort) with specific software. Monthly series of nine years of power consumption are analyzed statistically and stationary thermal-energy calculation is made applying the IRAM regulations through KG-MOD model. Empirical values are compared with the theoretical obtained.

Keywords: School building; Energy evaluation; Hygrothermal comfort. 


\section{Introducción}

El edificio escolar juega un papel importante en el bienestar general de estudiantes y profesores, y en el logro de los objetivos educativos. Un creciente número de investigaciones muestra que la calidad del aire interior, la ventilación, el confort térmico, la iluminación, la acústica, la calidad espacial y constructiva de la edificación y la estética, pueden afectar positiva o negativamente el desempeño de los alumnos y la moral de los docentes (USGBC, 2013).

Becker et al. (2007) de Israel, mencionan que en climas cálidos y templados, las grandes fuentes de calor internas que normalmente se encuentran en los edificios escolares impiden alcanzar el confort térmico sin refrigeración activa en verano, pero no son suficientes para eliminar la necesidad de calefacción en invierno. Afirman también que el uso del aire acondicionado no mejora la calidad del aire, y que la ventilación natural provoca pérdidas de energía no controladas. Constatan además que en las aulas -espacios cuya característica más dominante es su alta densidad de ocupación- se registran una elevada ganancia por fuentes de calor interno (5 kW), altos valores de CO2, vapor de agua y olores corporales, exponiendo a los jóvenes durante mucho tiempo a una baja calidad del aire en interiores, en el período más importante de sus años de crecimiento.

Estudios experimentales que relacionan el rendimiento escolar en función del confort térmico, demuestran que los alumnos obtienen mejores resultados en aulas acondicionadas térmicamente. Así, Pepler y Warner (1968) comprobaron que la tasa de error fue mayor a los $17^{\circ} \mathrm{C}$ y más baja a los $27^{\circ} \mathrm{C}$, con una diferencia de 20\% aproximadamente; sin embargo, el trabajo fue más lento. Las investigaciones de Wargocki y Wyon (2006) indican que a $20^{\circ} \mathrm{C}$ se logran óptimos resultados de los aprendices para distintas actividades prefijadas, y disminuye un $30 \%$ para temperaturas de $27^{\circ} \mathrm{C}$ a $30^{\circ} \mathrm{C}$. Los estudios mencionados, permiten reconocer que existe estrecha relación entre los niveles térmicos interiores y el rendimiento de los alumnos, destacando la importancia de establecer condiciones adecuadas para el desarrollo de un correcto proceso de enseñanza-aprendizaje.

Por otro lado, el análisis de consumos energéticos en tipologías escolares convencionales a partir de auditorías energéticas (registros de consumos tomados in situ, mediciones higrotérmicas y lumínicas de los espacios interiores) y de datos teóricos surgidos del cálculo de la demanda energética de dichos edificios, es un tema que ha interesado en la últimas décadas a numerosos grupos de investigación en diferentes partes del mundo (Desideri y Proietti, 2002; Dascalaki y Sermpetzoglou, 2011) y también de nuestro país (Gonzalo y Nota, 1999; San Juan, Hoses y González, 2000; Boutet, Hernández, Jacobo, et al., 2011). En La Pampa, se han diseñado y construido edificios para la educación, cuyas pautas de diseño estaban orientadas a lograr una mayor eficiencia energética (Filippín, 2005). El comportamiento térmico-energético de estos edificios escolares fue evaluado mediante monitoreos en distintos períodos del año, detectando diferentes temperaturas de confort según el tipo y localización del establecimiento.

Hasta el presente, no se ha registrado este tipo de información sobre escuelas existentes en la ciudad de San juan. Por ello, el objetivo de la actual investigación es evaluar el comportamiento higrotérmico y energético durante la crítica temporada estival sanjuanina, de un edificio escolar de tipología en peine construido post-terremoto 1944, seleccionado como caso de estudio; y conocer las condiciones de confort térmico de aulas específicas, durante los períodos monitoreados con la escuela en situación normal de ocupación.

\section{Metodología}

El estudio se basa en el procesamiento y análisis de datos empíricos de los ambientes interiores y datos teóricos calculados en función de las características arquitectónicas y de sus usuarios. La evaluación de la envolvente del edificio en régimen estacionario se efectúa de manera teórica, aplicando los procedimientos 
de cálculo sugeridos en las Normas IRAM 11601 (2002), 11603 (2012), 11604 (2001), 11605 (1996), 11659-1 (2004) y 11659-2 (2007). Se utiliza el modelo KG-MOD (Blasco Lucas, 2013), que calcula simultáneamente, la Transmitancia Térmica (K) de los distintos componentes de la envolvente, la Carga Térmica de Calefacción anual (Q), el Coeficiente Volumétrico de Refrigeración (GR), la Carga Térmica de Refrigeración (QR), y los correspondientes Factores de Forma FF y FAEP (Esteves et al., 1997). Dichos valores se comparan con los máximos admisibles recomendados para un nivel medio de calidad constructiva (B) por las Normas IRAM: 11604 (2001) para las cargas de calefacción, la 11659-2 (2007) para las cargas de refrigeración, la 11605 (1996) para el K y la 11601 (2002) para las resistencias superficiales.

Las mediciones se realizaron en la época estival, durante tres períodos de 14 a 24 días corridos, con el edificio en distintas situaciones de uso (Tabla 1), que permitan conocer cómo funciona en cada una de ellas. Para el monitoreo se utilizaron data-loggers marca $\mathrm{HOBO}$, con registros de temperatura $\left({ }^{\circ} \mathrm{C}\right)$ y humedad relativa (\%) en intervalos de 15 minutos. Los datos climáticos exteriores de temperatura y humedad se obtuvieron con una estación meteorológica marca DAVIS localizada en el Instituto de Energía Eléctrica (IEE) de la UNSJ (Pontoriero y Hoesé, 2013-2014). El procesamiento y el análisis comparativo de datos empíricos de temperatura y humedad recolectados durante las mediciones se lleva a cabo con el PROMEDI-HTL-V3 (Blasco Lucas, 2013). Las gráficas obtenidas permiten conocer el comportamiento higrotérmico de los ambientes interiores monitoreados.

Se relevan diariamente los registros de los contadores de electricidad durante los períodos monitoreados y se relaciona el consumo diario con las medias diarias de temperatura exterior y de tres aulas seleccionadas por su diferente ubicación y orientación (4, 5 y 6). Estos últimos datos se obtienen de las mediciones mediante programación de macros. Para realizar el análisis de las series de consumo eléctrico mensual correspondientes a 8 años de consumo eléctrico (2006-2014), se aplican técnicas estadísticas. Con los valores obtenidos se calculan los índices de consumo por unidad de superficie y se comparan con estándares internacionales y nacionales. Adicionalmente se estudian los niveles de confort en dichas aulas; para el análisis se emplea el programa para el cálculo de situación de confort PMV (Valor Medio Predicho) y PPD (Porcentaje de Personas en Disconfort) de Gonzalo (2003), que fue desarrollado, con ampliaciones y correcciones, según el procedimiento indicado en Santamouris y Asimakopoulos (1996); los índices se basan en los trabajos de Fanger (1982) y se usan en la Norma ISO 7730 (2005) para la evaluación del confort térmico en un ambiente. Se adoptan para los parámetros de cálculo los valores detallados en la Tabla 2.

\section{Características del edificio escolar}

La Escuela Teniente Pedro Nolasco Fonseca (EF), construida en el año 1953, pertenece históricamente al período de reconstrucción que tuvo la ciudad de San Juan luego del terremoto de 1944, formando parte a nivel nacional, del Plan Quinquenal Ilevado a cabo en el Gobierno de J. D. Perón. Su tipología edilicia es de "Lineal en Peine", muy característica de esta época y ampliamente utilizada en todo el país para resolver la arquitectura escolar. En la ciudad de

Tabla 1. Períodos de medición y estado de ocupación en el edificio.PMV TPPD.

\begin{tabular}{|c|c|c|c|c|c|c|c|c|}
\hline \multirow{2}{*}{ Período } & \multicolumn{2}{|c|}{ Fecha } & \multicolumn{3}{|c|}{ Cantidad } & \multirow{2}{*}{$\begin{array}{c}\text { Actividad } \\
\text { escolar }\end{array}$} & \multirow{2}{*}{$\begin{array}{c}\text { Ocupantes } \\
\text { en el edificio }\end{array}$} & \multirow{2}{*}{$\begin{array}{c}\text { Climatización } \\
\text { Mecánica }\end{array}$} \\
\hline & Inicio & Final & Dias & Horas & Registros & & & \\
\hline 1 & $01 / 11 / 2013$ & $15 / 11 / 2013$ & 14 & 336 & 1344 & Con & Con & Con \\
\hline 2 & $01 / 01 / 2014$ & $21 / 01 / 2014$ & 20 & 480 & 1920 & $\operatorname{Sin}$ & Sin & $\operatorname{Sin}$ \\
\hline 3 & $27 / 03 / 2014$ & $20 / 04 / 2014$ & 24 & 576 & 2304 & Con & Con & Sin \\
\hline TOTAL & $01 / 11 / 2013$ & $20 / 04 / 2014$ & 58 & 1392 & 5568 & ----- & - ---- & - --- \\
\hline
\end{tabular}

Fuente: Elaboración propia 
Tabla 2. Datos utilizados para el cálculo de los índices PMV y PPD

\begin{tabular}{|c|c|c|c|c|c|c|c|c|}
\hline \multirow[b]{2}{*}{ Aula } & \multicolumn{3}{|c|}{ Datos anatómicos } & \multirow{2}{*}{$\begin{array}{l}\text { Actividad } \\
\text { metabólica } \\
\text { (MET) }\end{array}$} & \multirow{2}{*}{$\begin{array}{c}\text { Nivel de } \\
\text { vest. NOV } \\
\text { (Clo) }\end{array}$} & \multirow{2}{*}{$\begin{array}{c}\text { Nivel de } \\
\text { vest. ABRIL } \\
\text { (Clo) }\end{array}$} & \multicolumn{2}{|c|}{ Velocidad del aire $(\mathrm{m} / \mathrm{s})$} \\
\hline & $\begin{array}{c}\text { Edad } \\
\text { (años) }\end{array}$ & $\begin{array}{c}\text { Peso } \\
(\mathrm{Kg})(*)\end{array}$ & $\begin{array}{c}\text { Talla (cm) } \\
\left({ }^{*}\right)\end{array}$ & & & & $\begin{array}{c}\text { Días } \\
\text { laborales }\end{array}$ & $\begin{array}{r}\text { Días no } \\
\text { laborales }\end{array}$ \\
\hline 4 & 9 & 27,5 & 132 & 70 & 0,6 & 0,8 & \multirow{3}{*}{0,15} & \multirow{3}{*}{0,10} \\
\hline 5 & 10 & 31,5 & 141 & 70 & 0,6 & 0,8 & & \\
\hline 6 & 11 & 33,0 & 155 & 70 & 0,6 & 0,8 & & \\
\hline
\end{tabular}

Fuente : Tabla realizada por las autoras. Los valores (*) corresponden a la Organización Mundial de la Salud.

San Juan se erigen 12 edificios con la misma. Este periodo histórico fue seleccionado por haber presentado altos índices de construcción de edificios escolares que permitieron elevar la matrícula de alumnos. El edificio está localizado en un medio urbano (Figura 1), inserto en un terreno amplio de 1 hectárea aproximadamente. Su perímetro limita al Este con la Avenida de Circunvalación (una arteria de alta velocidad de la ciudad de San Juan), al Oeste con la Calle Del Bono y hacia el Norte y Sur con un sector residencial de baja densidad.

Características de uso: La Escuela seleccionada es una institución de gestión Estatal que posee Niveles Inicial y Primario; se encuentra abierta de lunes a viernes de 7.30hs a 18hs con actividad de los encargados de la limpieza, y cuenta con dos turnos de clases, el de la mañana comprendido entre las $8 \mathrm{hs}$ y las $12 \mathrm{hs}$, y el de la tarde desde las $13.15 \mathrm{hs}$ hasta las $17.15 \mathrm{hs}$. La cantidad de alumnos es de 311 para la escuela primaria y de 90 para el Nivel Inicial; el personal docente, directivos y de limpieza asciende a 28 personas.

Clima: La ciudad de San Juan posee un clima árido seco con grandes amplitudes térmicas diarias y estacionarias (Tabla 3); está ubicada en la Región Cuyana al Centro Oeste de la Argentina, a los $31^{\circ} 6^{\prime}$ Latitud Sur y $68^{\circ} 5^{\prime}$ Longitud Oeste, a 615m-snm. La ciudad pertenece a la Zona bioambiental III (Templada Cálida), Subzona "a", con $896^{\circ} \mathrm{C}\left(\mathrm{GD}-18^{\circ} \mathrm{C}\right)$ y $1275^{\circ} \mathrm{C}$ (GD$20^{\circ} \mathrm{C}$ ), según la clasificación de la Norma IRAM 11603 (2012) para la República Argentina.

Características Edilicias: La organización funcional del edificio, se caracteriza por contar con una amplia circulación lineal principal, a la cual dan las puertas de la mayoría de las aulas, y se la aprovecha como SUM en ocasiones especiales (Figura 2). En las naves laterales, perpendiculares a la circulación principal, se ubican, en una de ellas servicios (cocina y sanitarios), y en la otra, aulas orientadas hacia el frente. El patio de ceremonia que se genera, es un espacio contenido y de fácil conexión con el interior. La escuela posee una superficie útil de $857 \mathrm{~m} 2$, con un Volumen de Masa Relativo de 13,8\% y una superficie Relativa de Aberturas de 16,5\%. En la Tabla 4 se detallan los valores de las distintas variables arquitectónicas.

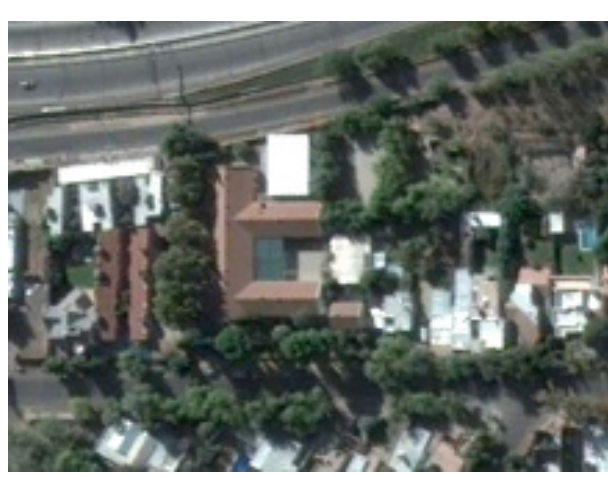

Fuente: Google Earth.

\begin{tabular}{|l|c|r|r|r|}
\hline \multirow{2}{*}{ PARAMETROS CuMATICOS } & \multirow{2}{*}{ UNIDAD } & \multicolumn{3}{|c|}{ VALOR } \\
\cline { 3 - 5 } & & \multicolumn{1}{|c|}{ ANUAL } & $\begin{array}{l}\text { INVIERNO } \\
\text { Julio }\end{array}$ & $\begin{array}{c}\text { VERANO } \\
\text { Enero }\end{array}$ \\
\hline T2 Media & \multicolumn{1}{c}{$\mathrm{C}$} & 18.2 & 8.4 & 27.1 \\
\hline To Media Máxima & \multicolumn{1}{c}{$9 \mathrm{C}$} & 27.1 & 18.1 & 35.2 \\
\hline To Media Mínima & \multicolumn{1}{c|}{$\mathrm{C}$} & 10.2 & -0.5 & 19.7 \\
\hline Humedad Relativa & $\%$ & 43.7 & 48.5 & 43.6 \\
\hline Amplitud Térmica Media & $9 \mathrm{C}$ & 17.1 & 18.6 & 15.5 \\
\hline $\begin{array}{l}\text { Radiación Media sobre } \\
\text { Superficie Horizontal }\end{array}$ & $\mathrm{kWh} / \mathrm{m}^{2}$ & 5.4 & 4.1 & 6.1 \\
\hline Velocidad Media Viento & $\mathrm{km} / \mathrm{h}$ & 13.3 & 11.0 & 16.3 \\
\hline
\end{tabular}

Fuente: www.tutiempo.net 

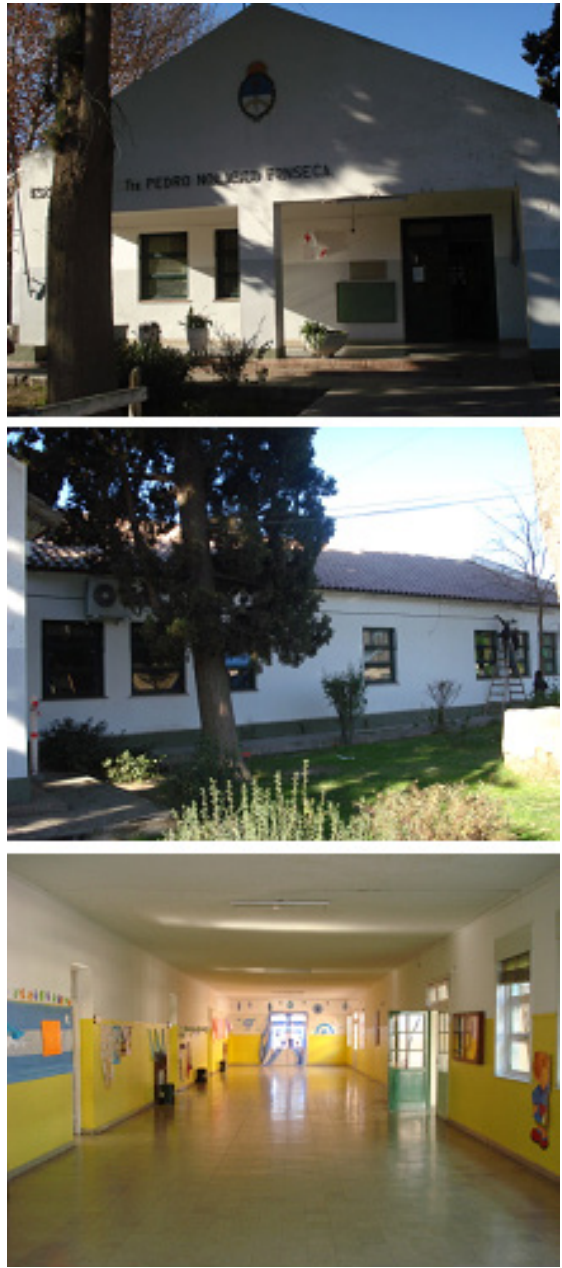

Fuente: Elaboración propia

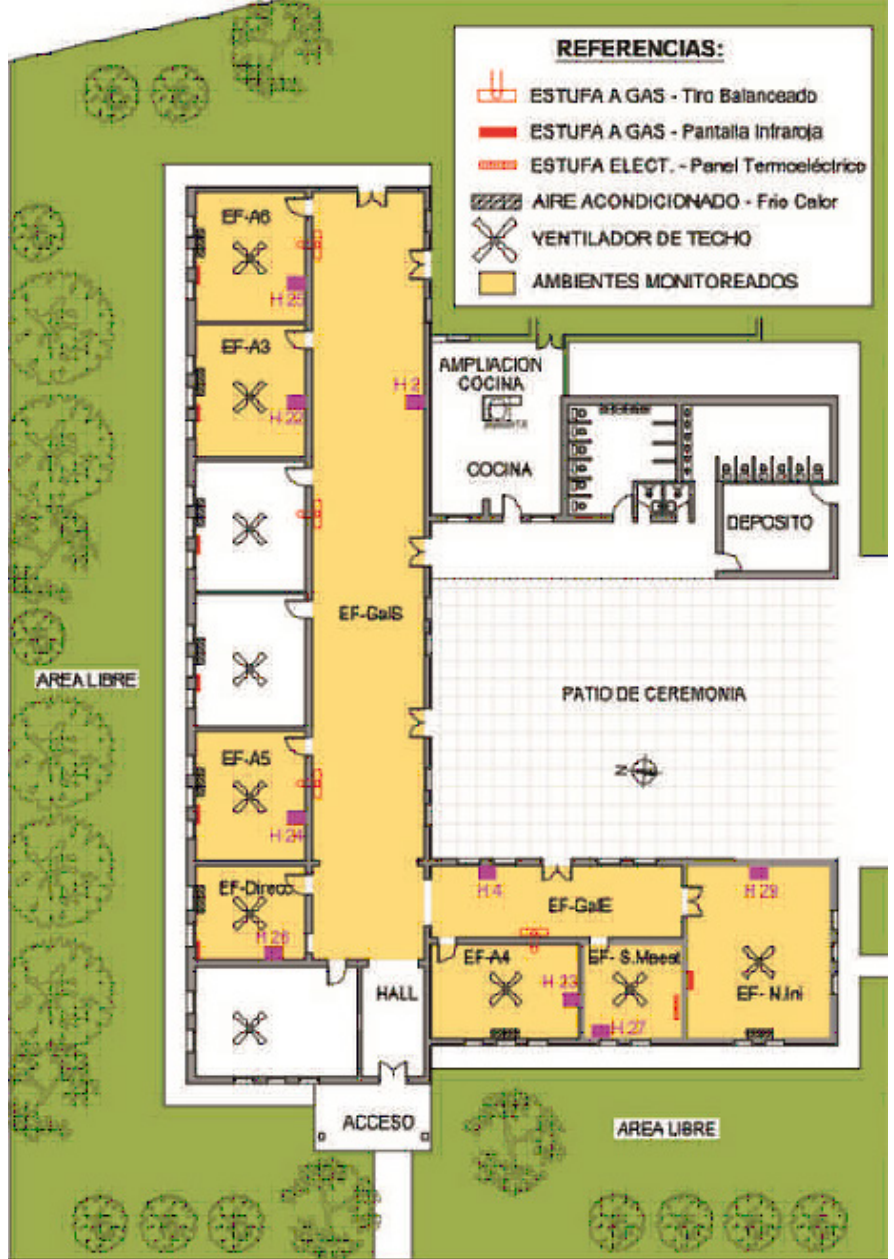

Fuente: Elaboración propia
Características de la tecnología constructiva: El edificio posee estructura sismorresistente, con muros de mampostería portante de ladriIlón con traba (34cm de espesor). El techo es inclinado con tirantes de madera y cubierta de tejas. El cielorraso suspendido genera un ático inaccesible. Conserva aún las ventanas y puertas originales, que son de carpintería de madera con vidrio simple.

Equipamiento: El equipamiento para climatización se encuentra indicado en el plano de la escuela (Figura 3), identificados con colores pertinentes. Para calefacción se utilizan estufas a gas tipo pantalla en las aulas, de tiro balanceado en las circulaciones cerradas y solo una estufa eléctrica en la sala de maestros. Cada aula y la dirección cuentan con equipos de aire acondicionado frio-calor y ventiladores de techo.

\section{Análisis térmico-energético estacionario}

Los resultados del análisis que permite conocer las propiedades térmicas de los componentes de la envolvente, se muestran en las Tablas 4 y 5 . En ellas se observa que el $\mathrm{K}$ de los mismos sobrepasa entre $47 \%$ y $341 \%$ los $\mathrm{K}$ max adm respectivos.

Para refrigeración, el cálculo de la carga térmica QR y el coeficiente volumétrico GR se llevó a cabo considerando las ganancias internas por el equipamiento, la iluminación, las personas y la radiación solar. Los valores obtenidos indican que el edificio se excede del QR adm y del GR adm con una diferencia negativa del $76 \%$ para ambos casos. La Figura 4 muestra gráficamente los datos de refrigeración en combinación con las características edilicias que más afectan el funcionamiento del edificio en verano. 
Tabla 4. Valores de características edilicias.

\begin{tabular}{|l|c|c|}
\hline \multicolumn{1}{|c|}{ CARACTERISTICAS EDILICLAS } & UNIDAD & VALOR \\
\hline Sup Cubierta: & $\mathrm{m}^{2}$ & 980 \\
\hline Sup Semicubierta: & $\mathrm{m}^{2}$ & 33 \\
\hline SUp Total: & $\mathrm{m}^{2}$ & 1013 \\
\hline Sup Util - Interior: & $\mathrm{m}^{2}$ & 857 \\
\hline FF & $\mathrm{m}^{-1}$ & 0.21 \\
\hline FAEP [Esteves, et al., 1997) & & 2.21 \\
\hline Sup. Rel. Aberturas (Puert.V Vent.) & $\%$ & 16.5 \\
\hline Vol. Masa Relativo & $\%$ & 13.80 \\
\hline Vol umen Interior & $\mathrm{m}^{3}$ & 3000 \\
\hline Carga Térmica de Calefacción Anual [Q] & $\mathrm{kWh}$ & 38862 \\
\hline Orientacion Preponderante & & Nor-Sur \\
\hline Orientacion Fachada Ppal. & Oeste \\
\hline Ventilación cruzada & SI \\
\hline
\end{tabular}

Figura 4.Comparación del Coeficientes $\mathrm{G}$ de refrigeración con valores admisibles y características edilicias.

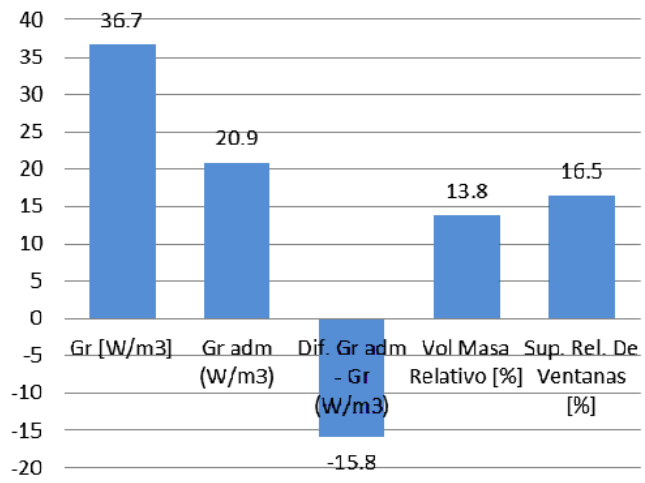

Fuente: Elaboración propia

\section{Monitoreo higrotérmico y consumos de energía} eléctrica

El monitoreo higrotérmico se realizó conforme lo detallado en la Tabla 1. En los planos de la Figura 3 se indican los espacios monitoreados, la ubicación de los sensores y el equipamiento para calefacción y refrescamiento que cuenta la escuela. El código de identificación de cada local monitoreado contiene la abreviatura del nombre de la Escuela (EF) seguido de su función (A3 = Aula 3). En base a expresiones de usuarios investigadas en trabajos previos ( $R e$ y Blasco, 2010) se adoptan como umbrales que definen la zona de confort el intervalo comprendido entre $20^{\circ} \mathrm{C}$ y $26^{\circ} \mathrm{C}$ para verano. Asimismo, se resaltan los fines de semana con recuadro color verde en las figuras correspondientes a consumo.

El análisis del período 1 se exhibe en la Figura 5 Izquierda, donde los espacios monitoreados permanecen en la zona de confort aproxima-
Tabla 5. Síntesis de los coeficientes K, Q y G.

\begin{tabular}{|c|c|c|c|c|}
\hline \multirow{3}{*}{$\begin{array}{l}\text { COMPONENTE } \\
\text { Y MATERLAL }\end{array}$} & \multicolumn{4}{|c|}{ VERANO } \\
\hline & \multirow{2}{*}{$\begin{array}{c}\text { K Max. } \\
\text { Adm. } \\
\text { Verano } \\
{\left[\mathrm{W} / \mathrm{m}^{2}{ }^{\circ} \mathrm{C}\right]}\end{array}$} & \multirow{2}{*}{$\begin{array}{l}\text { Tras. Térm. } \\
\text { K Verano } \\
\left.\mathrm{W} / \mathrm{m}^{20} \mathrm{C}\right]\end{array}$} & \multicolumn{2}{|c|}{$\begin{array}{c}\text { Dife rencia K } \\
\text { Verano }\end{array}$} \\
\hline & & & {$\left[\mathrm{W} / \mathrm{m}^{20} \mathrm{C}\right]$} & [36] \\
\hline $\begin{array}{l}\text { Muro: Labrillon } \\
\text { Exterior }\end{array}$ & 1.25 & 1.83 & -0.58 & -47 \\
\hline $\begin{array}{l}\text { Techo: madera y } \\
\text { tej as }\end{array}$ & 0.48 & 0.76 & -0.28 & -58 \\
\hline $\begin{array}{l}\text { Puerta: Madera y } \\
\text { Vidrio }\end{array}$ & 1.25 & 5.51 & -4.26 & -341 \\
\hline $\begin{array}{l}\text { Ventana: Madera } \\
\text { y Vidrio Simple }\end{array}$ & 1.25 & 4.73 & -3.48 & -279 \\
\hline \multirow{4}{*}{$\begin{array}{l}\text { CARGAS } \\
\text { TERMICAS }\end{array}$} & \multicolumn{4}{|c|}{ de Ref rigeración (QR) } \\
\hline & QR adm & $Q_{R}$ & \multicolumn{2}{|c|}{ Diferen cia $Q_{R}$} \\
\hline & {$[\mathrm{W}]$} & W] & {$[\mathrm{W}]$} & $\%$ \\
\hline & 62550 & 110067 & -47517 & -76 \\
\hline \multirow{4}{*}{$\begin{array}{l}\text { COEFICIENTES } \\
\text { VOLUMÉT RICOS }\end{array}$} & \multicolumn{4}{|c|}{ de Ref rigeración (GR) } \\
\hline & Gr adm & $\mathrm{G}_{R}$ & \multicolumn{2}{|c|}{ Diferencia $\mathrm{G}_{R}$} \\
\hline & W/m3] & {$[\mathrm{W} / \mathrm{mB}]$} & {$[\mathrm{W} / \mathrm{m} 3]$} & $\%$ \\
\hline & 20.85 & 36.69 & -15.84 & -76 \\
\hline Fuertas: & & & & \\
\hline \multicolumn{5}{|c|}{ K MaxAdn en Zanas II y N, caidad B canstruceiön-Norrma IRAM 11605 ( 1996} \\
\hline \multicolumn{5}{|c|}{ QR Admy GRAdm (fipo bloque) - IRAM $1859-2$ (2007) Tảiss 1B y2B } \\
\hline
\end{tabular}

Fuente: Elaboración propia

damente el $60 \%$ del tiempo, y en el interior del edificio la temperatura desciende alrededor de $10^{\circ} \mathrm{C}$ durante los días más calurosos. Esto sucede porque se accionaron los equipos de aire acondicionado y/o ventiladores en las aulas y en la dirección, de acuerdo a las preferencias de los usuarios. Lo cual demuestra la Figura 5 Derecha que relaciona el consumo eléctrico diario y las respectivas temperaturas medias exterior y de las aulas principales. En ellas se supera hasta $1^{\circ} \mathrm{C}$ el umbral superior de bienestar higrotérmico durante los 4 días más calurosos, pero se mantienen valores inferiores los restantes 10 días, fines de semana inclusive (sin climatización), demostrando que el edificio posee una significativa inercia térmica. Los espacios ubicados al Oeste tuvieron menores temperaturas máximas: EF-A4 $\left(28,4^{\circ} \mathrm{C}\right)$, EF-SMaest $\left(28,6^{\circ} \mathrm{C}\right)$ y EF-Nini $\left(28,2^{\circ} \mathrm{C}\right)$. Son ambientes con escasa ganancia solar directa que tiene lugar al finalizar la jornada escolar, durante unas pocas horas de la tarde, cuando recibe la sombra de la espesa arboleda situada en el frente de 
Figura 5. Edificio en condiciones reales de ocupación durante el Período 1. Izquierda: Dispersión higrotérmica. Derecha: Consumos diarios de energía eléctrica y temperatura exterior e interior en A4, A5 y A6.

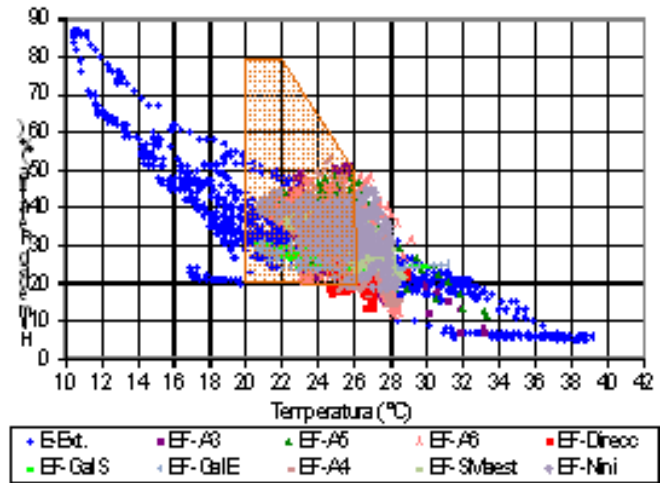

Fuente: Elaboración propia

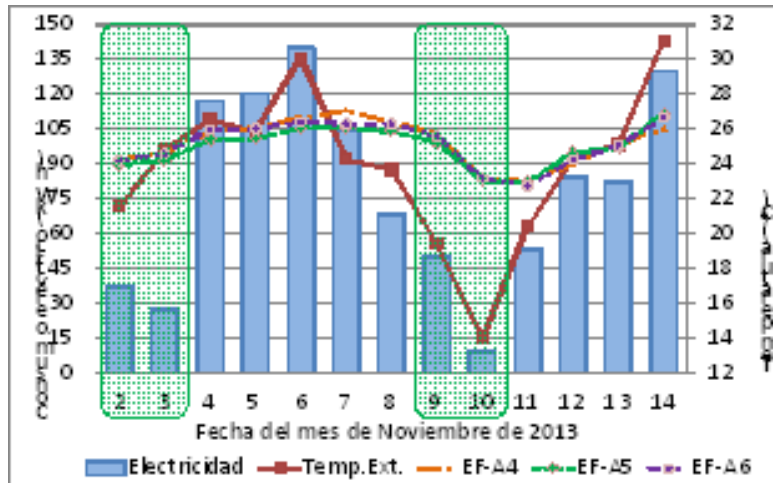

Fuente: Elaboración propia

Figura 6. Edificio en condiciones reales de ocupación durante el Período 2. Izquierda: Dispersión higrotérmica. Derecha: Consumos diarios de energía eléctrica y temperatura exterior e interior en A4, A5 y A6.



Fuente: Elaboración propia

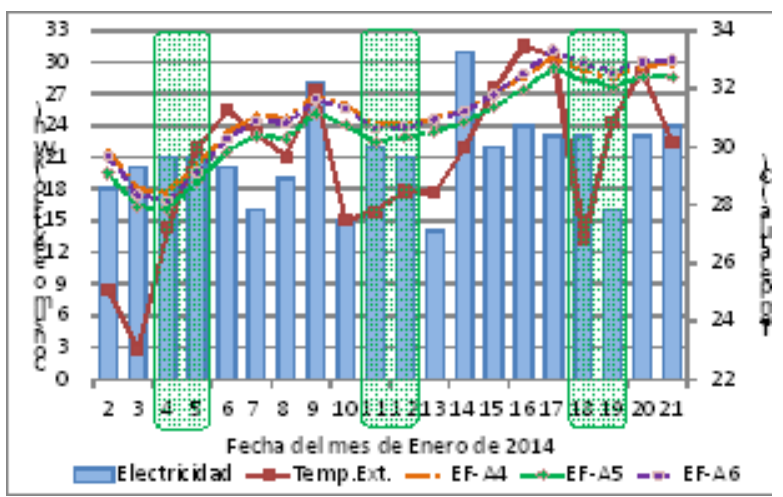

Fuente: Elaboración propia

del resto de los espacios oscilaron alrededor de los $30^{\circ} \mathrm{C}$. El consumo diario de energía eléctrica de este período permanece bajo, con escasas variaciones en torno al promedio $(21 \mathrm{kWh})$. Corresponde principalmente a la iluminación exterior del edificio que permanece encendida durante la noche e iluminación interior en los momentos de limpieza de cada aula. También el equipamiento de funcionamiento continuo, como la heladera, la bomba de la cisterna y el sistema de alarma.

El tercer período monitoreado corresponde a la finalización de la época estival y el inicio del otoño. En este intervalo la temperatura exterior varió entre los $18^{\circ} \mathrm{C}$ y los $26^{\circ} \mathrm{C}$ durante los primeros trece días, presentando un significativo descenso los 10 días restantes. A pesar de ello, 
Figura 7. Edificio en condiciones reales de ocupación durante el Período 3. Izquierda: Dispersión higrotérmica. Derecha: Consumos diarios de energía eléctrica y temperatura exterior e interior en A4, A5 y A6.

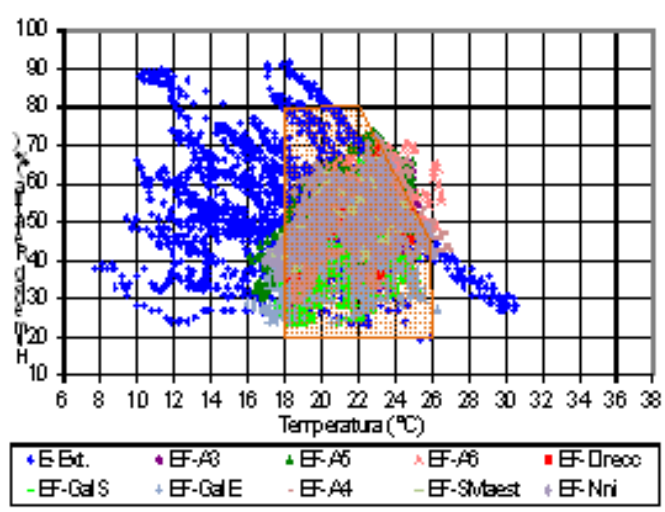

Fuente: Elaboración propia

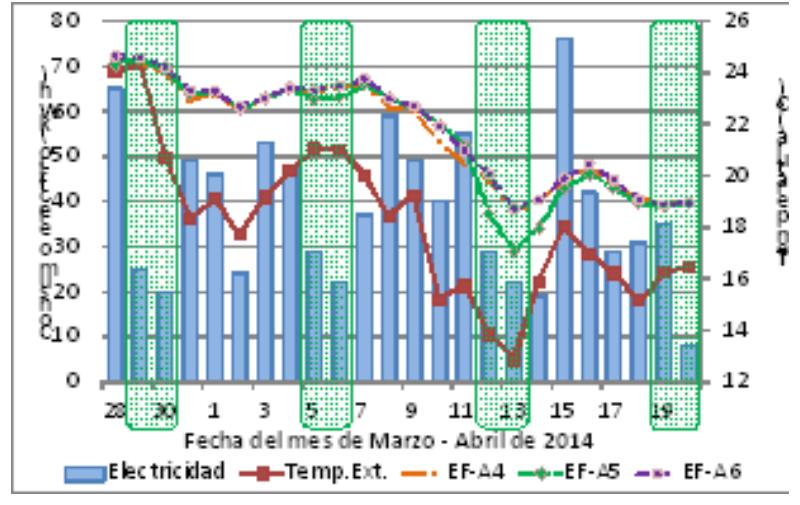

Fuente: Elaboración propia los ambientes interiores permanecieron todos dentro de la zona de confort (Figura 7), sin la utilización de equipos mecánicos de acondicionamiento. La mayor amplitud térmica del período se registró en la EF-GalE con $10,3^{\circ} \mathrm{C}$, seguida de EF-Nini con $9,2^{\circ} \mathrm{C}$ y de EF-A5 con $9,1^{\circ} \mathrm{C}$. Los espacios con mayor equilibrio y mejor comportamiento higrotérmico fueron EF-Direcc con variaciones entre $25,4^{\circ} \mathrm{C}$ y $18,1^{\circ} \mathrm{C}$, y EF-A3 donde osciló entre $25,2^{\circ} \mathrm{C}$ y $17,9^{\circ} \mathrm{C}$. EF-A4 presentó la mayor temperatura máxima $\left(26,9^{\circ} \mathrm{C}\right)$, cuando en el exterior se registraba $29,8^{\circ} \mathrm{C}$. El consumo eléctrico diario promedio durante fines de semana y feriado (02/04), cuando la escuela permaneció cerrada, fue de 23,8kWh similar a lo sucedido en enero (Figura 6). Esto se debe a que son los mismos artefactos de iluminación y equipamientos que se mantienen en funcionamiento. El día 28/3 tuvo un alto consumo de electricidad, producto de altas temperaturas, mientras que el día 15/4 se debió a una actividad extracurricular.

\section{Confort térmico en aulas}

En noviembre de 2013 los índices fueron calculados para los días más rigurosos ( $T^{\circ}$ máx exterior ? $38^{\circ} \mathrm{C}$ ) de todo el Período 1 (Tabla 1 ), entre el domingo 3/11 y el jueves 7/11 (Figura 8, Izquierda), con los datos de la Tabla 2. El aula 4 , orientada hacia el oeste con su envolvente sombreada por árboles muy densos, muestra que el mayor porcentaje de datos se encontra- rían dentro de un ambiente térmico en Bienestar Térmico (Gonzalo 2003) con valores de PMV entre $+0,5$ y -0,5 (entre Neutral y Ligeramente Cálido y Ligeramente Fresco de la Norma ISO 7730). En acuerdo con el índice PMV el PPD presenta valores menores al 10\%. Hacia el final del período analizado, con una temperatura promedio interior de $26,8^{\circ} \mathrm{C}$, el índice PMV se acerca al valor 1 , que corresponde a un Ambiente Térmicamente Aceptable según Gonzalo (2003), pero Ligeramente Cálido de acuerdo a la Norma. El Aula 5 orientada hacia el norte con un desvío de $10^{\circ}$ tiene una oscilación en los índices similar al caso anterior. El día 7 a las $15 \mathrm{~h}$ el valor de PMV es 0 indicando Bienestar Térmico, y neutral para la Norma ISO 7730 (2005), siendo la temperatura y la humedad relativa de $24,0^{\circ} \mathrm{C}$ y $34 \%$, respectivamente. El Aula 6, orientada al norte pero en el extremo de la crujía con mayor superficie en contacto con el exterior, exhibe oscilaciones bastante más marcadas que en los casos anteriores. Cuando el valor PMV está más próximo a 1, Ligeramente Cálido (Norma ISO 7730), el PPD se acerca al 13\% con una temperatura de aire interior y humedad relativa a las $13 \mathrm{hs}$ de $26^{\circ} \mathrm{C}$ y $40 \%$, respectivamente. La fluctuación diaria de los índices estaría mostrando que los equipos mecánicos de acondicionamiento térmico se encendieron alrededor de las 8, 11 y 13hs para ingresar a la zona de Bienestar Térmico. Sin cargas internas, el comportamiento de los índices en el día domingo es similar en las tres aulas, con pequeña variación en el aula 6 donde el PMV se aproxima a un ambiente Ligeramente Fresco, situación que 


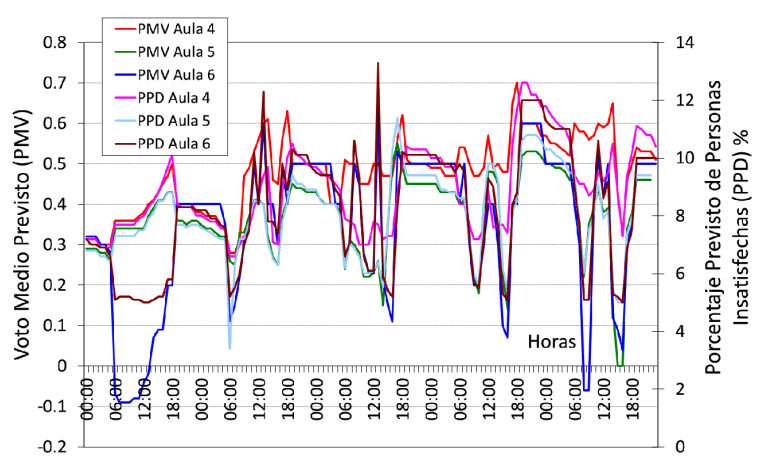

Fuente: Elaboración propia

estaría indicando que el equipo de acondicionamiento mecánico quedó encendido. Este día, las curvas diarias, como es de esperar, no presentan oscilaciones.

En el mes de abril de 2014 se realizan las estimaciones para 7 días, entre el 29/03 (sábado) y el 4/04 (viernes) con la situación del período 3 (Tabla 1) y los datos de la Tabla 2. En la Figura 8 (Derecha) se observa que en las tres aulas durante los días sábado y domingo el PMV oscila entre 0,2 y 0,6, valores que definirían el ambiente térmico como en Bienestar y Térmicamente Aceptable, respectivamente. Con el inicio de las actividades en el día lunes, tienen lugar las variaciones propias del uso de las áreas funcionales, en las cuales el PMV desciende hasta - 0,4 valorado como Térmicamente Aceptable (GonzaIo, 2003) y como Ambiente Ligeramente Fresco según la Norma ISO 7730 (2005). El día 2 de abril -sin actividad en el edificio- no existen las variaciones en los índices que nuevamente surgen durante jueves y viernes con una valoración de Bienestar Térmico (Gonzalo, 2003). El porcentaje previsto de personas insatisfechas es inferior al $10 \%$ excepto a las 7,8 y 9 hs del día 1 de abril con un valor de PPD de 11\% (PMV = -0,53).

\section{Consumos energéticos históricos}

La Figura 9 muestra datos de consumos históricos de electricidad, desde el año 2006 hasta el 2014, tanto en valores medios mensuales (Izquierda), como anuales y estacionales (Dere-



Fuente: Elaboración propia

cha). Se observa que hubo variaciones de consumo importantes durante el período del ciclo lectivo (de marzo a noviembre) y en forma bastante aleatoria entre 540kWh (marzo 2008) y 2297kWh (junio 2014), mientras que los valores promedio oscilaron entre $1060 \mathrm{kWh}$ y $1449 \mathrm{kWh}$. Es notorio el crecimiento del consumo en los meses de agosto a noviembre durante los años 2009, 2013 y 2014; como también en los meses mayo a agosto de los años 2010, 2012 y 2014, lo cual indicaría que se utiliza electricidad para la climatización interior incluso en el período invernal. La curva de regresión lineal del consumo anual muestra una tendencia creciente, al igual que las estacionales, entre las cuales la correspondiente a invierno tiene una pendiente levemente mayor que la de verano, mientras que la de menor pendiente es la curva de las temporadas intermedias (otoño y primavera, denominadas aquí "Resto" en forma unificada), $y$ todas presentan un buen nivel de ajuste con un coeficiente $\mathrm{R} 2$ razonable.

\section{Conclusiones}

La evaluación higrotérmica a partir de monitoreos, en tres períodos de época estival y con diferentes situaciones de uso, llevó a concluir que aun cuando el edificio posee gran inercia térmica, los usuarios requieren climatización artificial para alcanzar las condiciones adecuadas de bienestar. El consumo eléctrico asociado a este fin presenta una tendencia creciente a lo largo de los 9 años de registros históricos analizados. El índice de consumo eléctrico real por unidad de superficie para verano en los años 
Figura 9. Series de consumos eléctricos 2006-2014. Izquierda: Valores medios mensuales. Derecha: Valores medios estacionales y anuales.

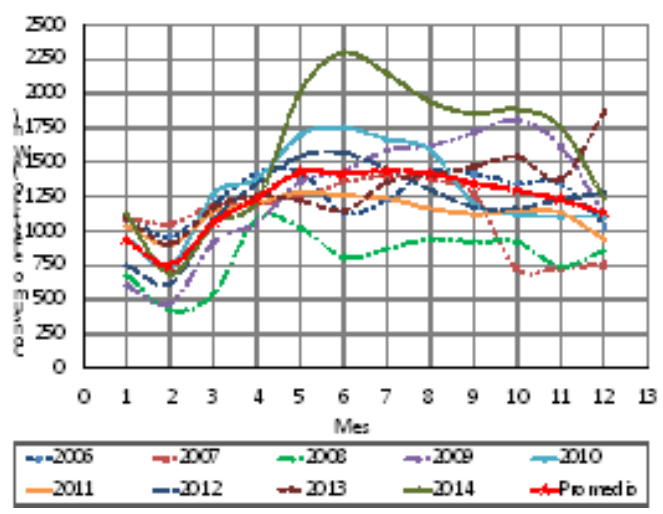

Fuente: Elaboración propia

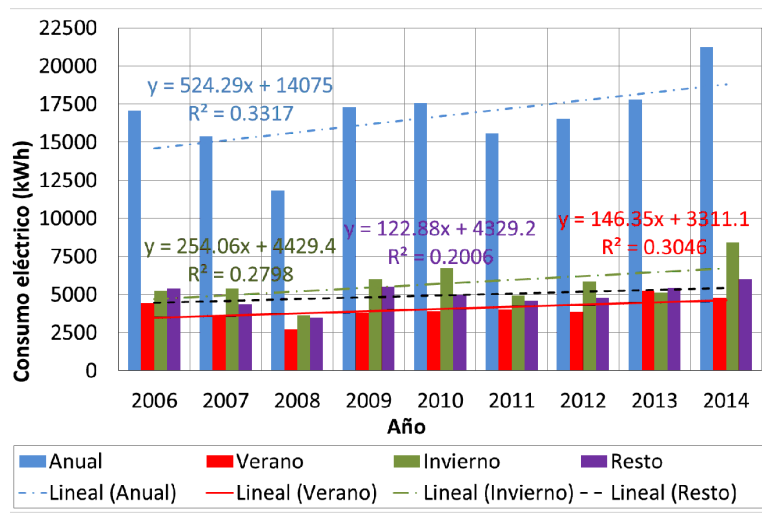

Fuente: Elaboración propia

vieron en confort. Para el mes de abril la temperatura media del aire interior desciende y oscila, en el turno mañana, entre los $22,9^{\circ} \mathrm{C}$ y $23,1^{\circ} \mathrm{C}$, con aumento de la humedad relativa promedio entre $52,6 \%$ y $53,9 \%$. En el turno tarde, los valores son de $23,5^{\circ} \mathrm{C}$ y $23,8^{\circ} \mathrm{C}(\mathrm{HR}=52,8 \%$ y $54,4 \%)$. Bajo estas condiciones y con un nivel de vestimenta más alto propio de la estación climática en estudio el índice PPD también es satisfactorio. El intervalo de temperaturas confortables para época estival a partir de este análisis, acuerda con la zona de confort delimitada en las figuras 4 a 6 de dispersión higrotérmica.

La época cálida, que para la ciudad de San Juan se extiende desde noviembre a marzo inclusive, se reconoce como la estación crítica que requiere principal atención para el acondicionamiento térmico. Queda previsto para futuros trabajos realizar la simulación del edificio y su calibración con datos medidos para analizar y mejorar la materialidad de la envolvente que permita cumplir con las recomendaciones de la normativa.

\section{Nota}

La información presentada forma parte del trabajo de Tesis doctoral de la Mag. Arq. María Guillermina Ré, del Doctorado en Arquitectura, FAUD, de la Universidad de Mendoza, desarrollada bajo la dirección de la Dra. Arq. Celina Filippín y la co-dirección de la Dra. Arq. Irene Blasco Lucas. 


\section{Bibliografía}

Becker, Rachel; Goldberger, Itamar; Paciuk, Mónica. (2007). Improving energy performance of school buildings while ensuring indoor air quality ventilation. Building \& Environment 42, pp. 3261-3276.

Blasco Lucas, Irene (2013). Arquitectura sustentable en hábitat rural de zona árido-sísmica: Aportes teórico-metodológicos. Tesis Doctoral en Arquitectura. Universidad de Mendoza.

Boutet, M.L.; Hernández, A.L.; Jacobo, G.J.; Martina P.E; Corace, J.J. (2011). Auditorías higrotérmicas y lumínicas de dos edificios escolares de nivel inicial de la ciudad de resistencia, en condiciones reales de ocupación. AVERMA, 15 p. 05.29 a 05.36

Dascalaki, Elena; Sermpetzoglou, Vasileios (2011) Energy performance and indoor environmental quality in Hellenic schools. Energy and Buildings 43, p. 718 a 727. Elsevier.

Desideri, U.; Proietti, S. (2002) Analysis of energy consumption in the high schools of a province in central Italy. Energy and Buildings 34, p. 1003 a 1016. Elsevier.

Esteves, A.; Gelardi, D.; Oliva, A. (1997). The shape in the bioclimatic architecture: the FAEP factor. Proceedings of II Conference on Teachers in Architecture, Chapter 3.12.

Fanger, P.O. (1982) Thermal comfort. R.E.Krieger Publishing Company, Malabar,FI.

Filippín, Celina (2005). Uso eficiente de la energía en edificios. Ed. Amerindia. Santa Rosa, La Pampa.

Gonzalo, Guillermo E. (2003) Manual de Arquitectura Bioclimática. Editorial Nobuko. Programa para el cálculo de situación de confort: PMV y PPD. CEEMA, IAA, FAU, UNT. Noviembre 2002

Gonzalo, Guillermo E.; Nota, Viviana M. (1999) Determinación de índices de consumos de energía para distintas funciones edilicias en el área de san miguel de Tucumán. AVERMA. Vol.3, Art. 08-45

IRAM (1996, 2001, 2002, 2004, 2007 y 2012). Serie 11600 de Normas para acondicionamiento térmico de edificios. Instituto Argentino de Normalización.

ISO 7730 (2005) Ergonomics of the thermal environment. Analytical determination and interpretation of thermal comfort using calculation of the PMV and PPD indices and local thermal comfort criteria. Third edition. Switzerland.

Pepler, R.D., Warner, R.E. (1968). Temperature and learning: an experimental study. ASHRAE Transactions, 74, p. 211219.

Pontoriero, Domingo; Hoesé, Liliana (2013-2014). Programa de relevamiento de datos meteorológicos de la ciudad de San Juan. Instituto de Energía Eléctrica, UNSJ.

Ré, M. Guillermina y Blasco Lucas, Irene (2010). Comportamiento higrotérmico, lumínico y energético de edificios residenciales ubicados en la ciudad de San Juan. Revista AVERMA, 4, Art 05-35.

San Juan G.; Hoses S.; González D. (2000) Sensibilidad de variables edilicias y energéticas de tipologías edilicias escolares en dos situaciones regionales de nuestro país. AVERMA. 4. Art 07-29

Santamouris M. and Asimakopoulos D, Editores (1996) Passive Cooling of Buildings. James \& James.

USGBC (2013). LEED Reference Guide V4. United States Green Building Council, Washington D. C. Disponible en: http://www.usgbc.org/leed/rating-systems/om 